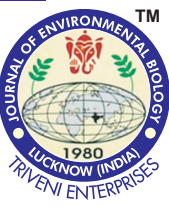

\title{
Growth estimation during hardening phase of tissue cultured banana plantlets using bootstrapped artificial neural network
}

\begin{tabular}{lll}
\hline Paper received: 19.06 .2018 & Revised received: $13.11 .2018 \quad$ Re-revised received: 24.12 .2018
\end{tabular}

\section{Authors Info}

S. Revathi', N. Sivakumaran ${ }^{1}$, D. Ramajayam ${ }^{2 *}$, M.S. Saraswathi', S. Backiyarani ${ }^{2}$ and S. Uma ${ }^{2}$

'Department of Instrumentation and Control Engineering, National Institute of Technology, Tiruchirappalli-620 015, India

${ }^{2}$ ICAR - National Research Centre for Banana, Tiruchirappalli-620 102, India

*Corresponding Author Email : ramajayamd@gmail.com

\section{Edited by \\ Dr. Nirmal Jeet Kaur}

\section{Reviewed by}

Dr. Hemant Ranjan

Dr. Kundan Kishor

\section{Abstract}

Aim : The study aims to develop an advanced non-destructive method to estimate the plant growth rate of tissue culture propagated banana plantlets during primary hardening phase inside the greenhouse using Bootstrapped Artificial Neural Network (BANN).

Methodology : Both non-destructive growth parameters like plant height, girth, number of leaves, leaf length and leaf breadth, and destructive growth parameters like number of roots, longest root length, fresh and dry weight were measured periodically on selected plants of one week to nine week old which were kept in greenhouse at ICAR-National Research Centre for banana. In addition to plant growth parameters, greenhouse temperature, radiation and carbon dioxide concentration were also recorded daily. The experimental data obtained using destructive measurements were recorded on a small sample of size $\mathrm{n}$, and hence re-sampling for bootstrap involves $n$ repeated trials of simple random sampling with replacement. These sets of bootstrap samples were finally used as input to develop neural model using a novel methodology of bootstrap re-sampling based artificial neural network (ANN) for studying the progress of plant ontogeny.

Results : The growth estimation analysis of plants in terms of its leaf area and biomass production was performed without physically handling the test plants using bootstrap ANN. The notion of prediction performance is validated through statistical indices namely Nash and Sutcliffe efficiency coefficient, root means square error and mean absolute error. The approximate estimates of mean relative growth and net assimilation rate of plants were 0.036 and 0.027 , and the corresponding variance were $1.5 \times 10^{-6}$ and $2.12 \mathrm{x}$ $10^{-6}$, respectively.

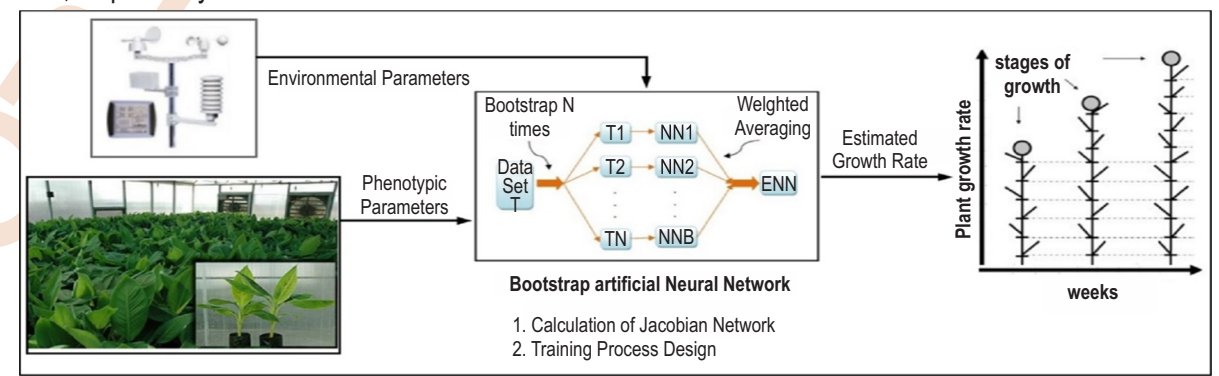

Interpretation : Based on the non-destructive plant growth observations, the measures to increase the overall plant growth can be significantly predicted well in advance. This projected plant growth statistics at an early stage of hardening serves as an essential component in planning and evaluation of investments on protected structure to improve the productivity and profitability of banana tissue culture industry.

Key words: Artificial neural network, Bootstrapped sample, Greenhouse technology, Tissue culture banana

How to cite : Revathi, S., N. Sivakumaran, D. Ramajayam, M.S. Saraswathi, S. Backiyarani and S. Uma: Growth estimation during hardening phase of tissue cultured banana plantlets using bootstrapped artificial neural network. J. Environ. Biol., 40, 719-724 (2019). 


\section{Introduction}

Greenhouse is essential for production of superior quality banana plantlets round the year. It protects banana plantlets against rain, frost, storms, any sudden infestation of pests, pathogens, excessive moisture, temperature, etc. Tissue cultured plants are highly sensitive hence they are grown under protected structures (greenhouse) which enable control of environmental variables. It has been reported that plant growth and production is at least ten times higher in greenhouse than in open field (Max, 2012). Tissue-cultured banana plantlets are grown in vitro under low-light and temperature with high humidity. During the last phase of micropropagation, steps are taken to grow individual banana plantlets and prepare them for acclimatization under greenhouse condition. Chandra et al. (2010) mentioned the reduction of mortality during the acclimatization or hardening stage under greenhouse which takes 7-10 weeks. According to a study conducted by Perez and Hooks (2008), the plants are generally allowed to acclimatize in greenhouse for about two months to attain about 20 to $30 \mathrm{~cm}$ height before they are transplanted in the fields. This period allows for physiological adaptation of plant to the changes in the climate or environment, such as light, temperature or humidity.

In a developing country like India, monitoring of plant growth is essential for reliable prediction of crop yields at initial stages of crop growth which will facilitate effective utilization of growth strategies. Soil water balance modeling by linking soil and plant models was pioneered by Slatyer and Taylor (1960) and later Pinter et al. (2003) developed crop models using remote sensing to obtain strategic crop forecasts for open field cultivation. But this approach lacked a comprehensive quantitatively analyzed precise estimate owing to absence of biophysical crop model. However, in the case of greenhouse structure based cultivation, the environment inside the closed structure, plants under cultivation and their interactions are the main components of the ecosystem and measurement of growth rate is necessary for estimating the crop yield (Albright et al. 2001). In earlier studies, researchers have critically emphasized the measurement of eco-physiological parameters that benefit to foresee the yield at an early stage of hardening which drives as a factor to estimate the net assimilation rate non-destructively. (Kishor Kumar et al., 2017; Pompelli et al., 2012; Sezer et al., 2009; Sone et al., 2009). Non-destructive estimation of phenotypic data offers reliable and inexpensive alternatives to determine several growth indices in banana (Potdar and Pawar, 1991), chestnut (Serdar and Demirsoy, 2006), maize (Mokhtarpour et al., 2010) and tomato (Ronga et al., 2017). Simon (1999) has detailed many neural network architectures and applications which serve as a comprehensive foundation. Priddy and Keller (2005) explained the methodology of dealing with limited number of data. Bootstrap re-sampling method is one such effective technique which generates new data sets using Monte Carlo technique. Despite all the recent interest in nonparametric network analysis, applications of bootstrap methodology remain virtually unexplored. Efron and Tibshirani
(1993) invented this accurate way of dealing with small sample size and it is defined as a computational procedure that uses intensive re-sampling with replacement to reduce uncertainty under limited data condition. The unbiased artificial neural network (ANN) ensemble model is derived by aggregating individual ANN model outputs. By the estimates of the potential ANN model outputs, the assessment of crop growth is accomplished. These net assimilation estimates also serves to manage, control and optimize plant growth effectively. A non-destructive and inexpensive method for measuring of the plant growth rate is essential. It is extremely important that such model be reliable and accurate. Artificial neural network (ANN) is one such method which is increasingly used in estimation studies. The purpose of the present study was to develop a predictive model without destructing the primary hardened banana plantlets grown inside the greenhouse environment by using advanced ANN concepts for the estimation of their growth rate parameters.

\section{Materials and Methods}

Experimental greenhouse structure: Data was collected from greenhouse in ICAR-National Research Centre for Banana, Tiruchirapalli, Tamil Nadu. The tissue cultured banana seedlings were kept in the greenhouse structure having a length of $8 \mathrm{~m}$, width of $6 \mathrm{~m}$ and covered with carbonate sheets to provide proper sunlight to plants. To maintain the required temperature and humidity inside, it is provided with the fan-pad cooling system. One end of the greenhouse wall is equipped with $5 \mathrm{~cm}$ aspen pad arrangement with a maximum $21 \mathrm{~g} \cdot \mathrm{H}_{2} \mathrm{O} \cdot \mathrm{Sec}^{-1}$ of flow of water and the opposite end of the wall is fitted with two suction fans of capacity $230 \mathrm{~V}, 50 \mathrm{~Hz}, 0.5 \mathrm{HP}$ belt type driven by induction motor with a maximum ventilation rate of $9.6 \mathrm{~m} \mathrm{sec}^{-1}$.

Periodic growth measurements: Plant growth parameters such as plant height $(\mathrm{cm})$, girth $(\mathrm{cm})$, number of leaves and roots, length of longest root $(\mathrm{cm})$, leaf length $(\mathrm{cm})$ and width $(\mathrm{cm})$ were measured on randomly selected five plants till nine weeks at weekly interval. Also, the data on greenhouse temperature, radiation and carbon dioxide concentration were consecutively collected in an hourly basis for during hardening phase from January to March, 2018. This limited dataset serves as a primary input and output to the neural network.

Bootstrap re-sampling method: The methodology invented by Efron (1979) was used for bootstrap re-sampling as it is more accurate for dealing small sample size dataset. The methodology involves creating a large number of new samples from the original data, re-sampling the available data to build a sampling distribution. This assumes that the sample distribution is representative of the entire population. A Monte Carlo technique, randomly generating new data sets to simulate the process of data generation, accomplishes this re-sampling. Here, $n$ size original data which is obtained from destructive experiment is resampled to obtain B bootstrap re-samplings. A bootstrap set then forms the data used to train a neural network which results in $B$ neural network models. 
Bootstrap ANN model-based crop phenotypic model: The mean and standard deviation values of zero-mean normalized bootstrap re-samplings obtained using bootstrap method was partitioned as $70 \%$ and $30 \%$. The former was used as training set while later was used as validation set to examine the model generalization. Training set was used to train the model to estimate the model parameter; testing data was used to test the generalization capability of the model.

Estimation of plant growth: The relative growth rate $(R)$ and net assimilation rate $(E)$ were computed, respectively, using $R=(1 / W)(d W / d t)$ and $E=(1 / L A)(d W / d t)$, where $W$ is the dry weight of plant at time tand LA is leaf area. Similarly, mean

$$
\bar{R}=\frac{\left(\log _{e} W_{2}-\log _{e} W_{1}\right)}{t_{2}-t_{1}} \text { and } \bar{E}=\left\{\frac{\left(W_{2}-W_{1}\right)\left(\log _{e} L_{2}-\log _{e} L_{1}\right)}{\left\{\left(L_{2}-L_{1}\right)\left(t_{2}-t_{1}\right)\right\}}\right\}
$$

were calculated, where $t_{1}$ and $t_{2}$ are the period between the measurements and $W_{1}, W_{2}, L_{1}$ and $L_{2}$ are their respective plant dry weights and leaf areas measurements over the period.

A set of growth data were derived using bootstrap resampling method as follows; Let, there be $n$ replicate plants at each measurement and denote the dry weight as $W_{i j}$ and leaf area as $L_{i j}(j=1 \ldots n)$. Then for the $j^{\text {th }}$ replicate set of plants

$$
\bar{R}_{j}=\frac{\left(\log _{e} W_{2 j}-\log _{\mathrm{e}} W_{1 j}\right.}{t_{2}-t_{1}} \text { and } \bar{E}_{j}=\left\{\frac{\left(W_{2 j}-W_{1 j}\right)\left(\log _{e} L_{2 j}-\log _{e} L_{1 j}\right)}{\left\{\left(t_{2}-t_{1}\right)\left(L_{2 j}-L_{1 j}\right)\right\}}\right\}
$$

The expected values (E) of $R$ and $E$, respectively, is defined as the arithmetic mean of $R$ and $E$ across $n$ replicates as $\mathrm{E}(\bar{R})=(1 / n) \cdot \sum_{j=1}^{n} R_{j}$ and $(E)=(1 / n) \cdot \sum_{j=1}^{n} E_{j}$. Similarly, the variance of $R$ and $E$ are then calculated as $V(R)=\{1 /(n-1)\} \cdot \sum_{j=1}^{n}\{R j-E(R)\}^{2}$ and $V(E)=\{1 /(n-1)\} . \sum_{j=1}^{n}\{E j-E(E)\}^{2}$ respectively.

Performance measures: The performance of developed models is evaluated using Nash-Sutcliffe Efficiency (NSE) coefficient of simulation efficiency for validation of datasets by equation

$$
\mathrm{NSE}=\left[\frac{\sum_{i=1}^{n}\left(T_{i}-O_{i}\right)^{2}}{\sum_{i=1}^{n}\left(O_{i}-\bar{O}\right)^{2}}\right] \times 100
$$

where, $O_{i}$ and $T_{i}$ are the observed values through experiment and estimated values using neural model, 0 is the mean of observed values and $n$ is the total number of observations. The greater value of NSE indicates a strong relationship between the observed and model predicted values. Additional network performance measures were evaluated using the root mean square error $($ RMSE $\left.)=\sqrt{ }\left(1 / n \sum_{i=1}^{n} \mid T_{i}-O_{i}\right)^{2}\right)$, mean absolute error $(M A E)=1 / n$ $\left.\sum_{i=1}^{n}\left|T_{i}-O_{i}\right|\right)$. The lesser value of MAE and RMSE indicate a perfect match between the observed and estimated values.

\section{Results and Discussion}

Estimation of plant growth: Assimilation is the process by which crop plants convert carbon dioxide into plant biomass through photosynthesis and it is an important measure of crop ontogeny. The process is driven by the application of photosynthetic photon flux in the presence of carbon dioxide and appropriate temperature and nutrition. The various aspects of growth analysis can be well understood by measuring net assimilation rate over the growing period. It is understood that efficiency of banana leaf as measured by $E$ is affected by both internal plant demand and external climatic factors. Net assimilation rate helps in quantitative terms to help interpret crop yields under different environments. The average values of different phenotypic characters measured over the hardening periods on the tissue cultured banana plants kept in greenhouse is given in Table 1. Also, the net assimilation rate is simulated using greenhouse temperature, radiation and carbon dioxide concentration as shown in Fig. 2. This limited plant data set were used as a primary input and output to the neural network which coordinated well with the Bootstrap small sample properties listed by Scholz (2007). Song et al. (2013) and Fisher et al. (1991) also successfully applied similar bootstrap algorithms to such small sample data.

Bootstrap ANN model-based crop phenotypic model: The traditional way to study crop dry-matter partitioning pattern is to harvest and dry the plant materials. This technique is time consuming, parts of plants are destroyed, and the growth rate is adversely affected. Only limited data records are possible for modeling purpose since the periodic destructive measurements are performed on very less sample of plants during the growing

Table 1 : Phenotypic measurements of banana plant

\begin{tabular}{llllllll}
\hline Week & $\begin{array}{l}\text { Height } \\
(\mathbf{c m})\end{array}$ & $\begin{array}{l}\text { Girth } \\
(\mathbf{c m})\end{array}$ & $\begin{array}{l}\text { No of } \\
\text { leaves }\end{array}$ & $\begin{array}{l}\text { No of } \\
\text { roots }\end{array}$ & $\begin{array}{l}\text { Longest root } \\
\text { length }(\mathbf{c m})\end{array}$ & $\begin{array}{l}\text { Leaf length } \\
(\mathbf{c m})\end{array}$ & $\begin{array}{l}\text { Leaf breadth } \\
(\mathbf{c m})\end{array}$ \\
\hline 1 & 7.10 & 3.2 & 3.3 & 8 & 8.1 & 28 & 14 \\
2 & 8 & 3.2 & 3.6 & 8 & 9 & 28.5 & 14 \\
3 & 11.1 & 3.3 & 4 & 8 & 10.10 & 29 & 14 \\
4 & 12 & 4 & 4 & 8 & 11.10 & 29 & 14 \\
5 & 12 & 4 & 4 & 9 & 11.40 & 29.5 & 14.5 \\
6 & 13 & 4 & 4 & 9 & 12 & 30.5 & 14.5 \\
7 & 13 & 4 & 4.3 & 9 & 13 & 31 & 15 \\
8 & 14 & 4 & 4.3 & 10 & 14 & 31 & 15.5 \\
9 & 14 & 4 & 4.3 & 11 & 16 & 31.5 & 15.5 \\
\hline
\end{tabular}


Table 2 : Statistical values of normalized bootstrap resamplings

\begin{tabular}{llllllllll}
\hline $\begin{array}{l}\text { Statistical } \\
\text { index }\end{array}$ & Height & Girth & $\begin{array}{l}\text { No of } \\
\text { leaves }\end{array}$ & $\begin{array}{l}\text { No of } \\
\text { roots }\end{array}$ & $\begin{array}{l}\text { Longest } \\
\text { root length }\end{array}$ & $\begin{array}{l}\text { Leaf } \\
\text { length }\end{array}$ & $\begin{array}{l}\text { Leaf } \\
\text { breadth }\end{array}$ & $\begin{array}{l}\text { Dry } \\
\text { weight }\end{array}$ & $\begin{array}{l}\text { Leaf } \\
\text { area }\end{array}$ \\
\hline Mean & 0.2859 & 0.3447 & 0.3387 & 0.3939 & -0.0834 & 0.0178 & -.0815 & 0.0642 & 0.0985 \\
Standard & 0.2377 & 0.2771 & 0.2204 & 0.2176 & 0.1945 & 0.2475 & 0.2780 & 0.2240 & 0.2156 \\
deviation & & & & & & & & & \\
\hline
\end{tabular}
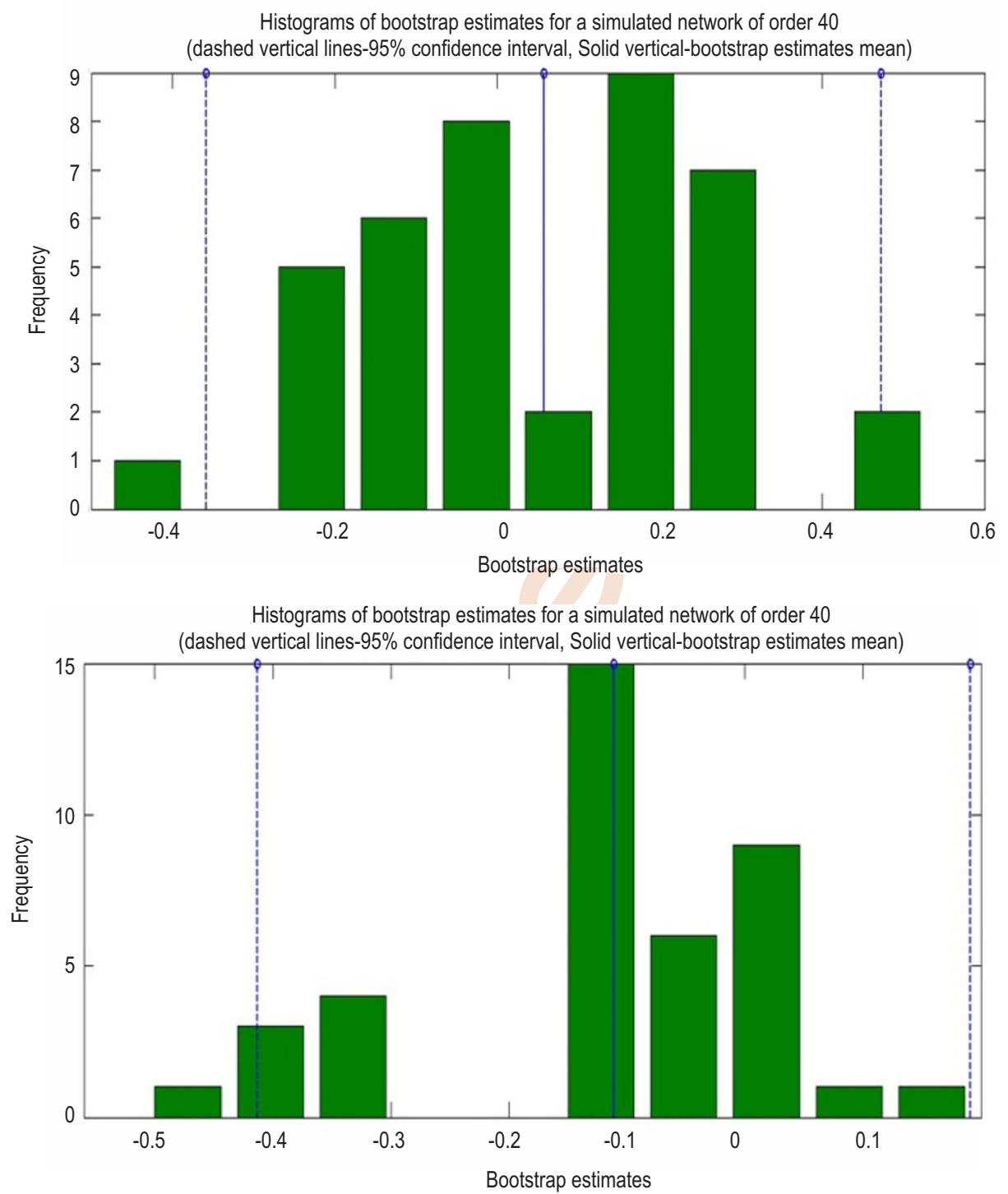

Fig. 1 : Histogram of bootstrap estimates of simulated network for dry weight and leaf area.

period inside the greenhouse. But for neural network modeling, a relatively large network is needed to estimate the leaf area and dry weight. Hence, bootstrap re-sampling technique is applied to the neural networks to estimate the predictive distribution for unseen inputs. In case of banana plant, the rate of fruit development is directly related to the number of functional leaves and leaf area. Therefore, a non-destructive and precise method for determination of leaf area and dry weight mass of banana is proposed by bootstrap-based ANN which measures the crop growth rate. Initially, the limited destructive measurements 


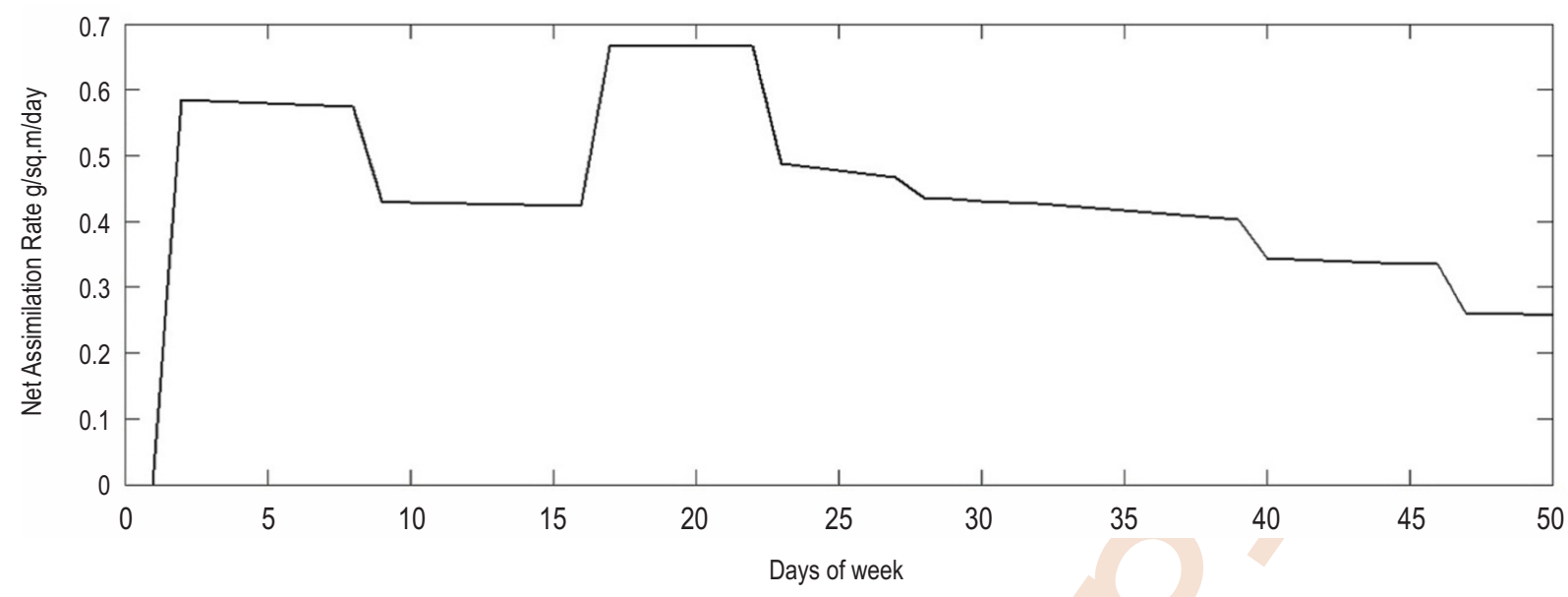

Fig. 2 : Net assimilation rate.

recorded on banana plantlets form initial dataset which are further resampled by bootstrapping method to establish a larger dataset for the purpose of modeling. Chernick and Labudde (2009) suggested that for small sample size the lower order bootstrap performs better than the higher order, and thus 50 bootstrap samples are used to represent the input dataset of neural network. The mean and the standard deviation values of zeromean normalized bootstrap re-samplings are given in Table 2 .

From modeling viewpoint, the ability of neural networks to deal with nonlinear systems is perhaps most significant. This promising possibility to provide nonlinear system models tiled to employ a nonlinear connectionist network to model dry weight and leaf area. For this purpose, the data from bootstrap method was portioned into $70 \%$ as training set and $30 \%$ as validation set to examine the model generalization. A number of results have been published previously in the similar line by Goodwin and Sin (1984), Cybenko (1988, 1989); Carrol and Dickinson (1989); Funahashi (1989) and Hornik et al. (1989) showing that a feed forward network of multilayer perception type can approximate arbitrarily well for a continuous function. Hence, output error model is chosen with ten neurons in the input layer and two neurons in the output neuron with linear transfer functions and using one hidden layer with hyperbolic tangent sigmoid transfer function. The sigmoid and tan $\mathrm{h}$ functions are similar: sigmoid ranges from 0 to 1 while tan $\mathrm{h}$ ranges from -1 to 1 . The histogram of bootstrap estimates of simulated network of order 40 is plotted in Fig. 1. Most of the estimates lie within the confidence interval which quantifies the uncertainty interval of output estimates. Thus, with basic nondestructive phenotypic measurements and greenhouse environment measurements, the aforementioned neural model approach can be used to estimate the biomass production and leaf area of banana seedlings without any periodic destructive sampling like air oven drying of the test plants.

Performance measures: The efficiency of neural network to estimate dry weight and leaf area obtained from nondestructive phenotypic measurements is exemplified using the criteria proposed by Nash and Sutcliffe (1970) to evaluate the performance of trained network. Lin et al. (2017) used NashSutcliffe Efficiency (NSE) coefficient in goodness-of-fit measures for daily runoff simulation with SWAT. In the present study, the performance of developed models was evaluated using this coefficient of simulation efficiency for validation datasets using NSE equation. Based on the values of root mean square error (RMSE), the mean absolute error (MAE) and Nash-Sutcliffe Efficiency (NSE), ANN model was found to be more accurate both in terms of training (Dry weight: RMSE $=1.18 \times 10^{-6}, \mathrm{MAE}=$ $7.74 \times 10^{-7}$ and NSE $=99.15$; Leaf area: RMSE $=0.0802$, MAE $=$ 0.0545 and NSE $=85.96$;) and testing phases (Dry weight $:$ RMSE $=0.5294, \mathrm{MAE}=0.466$ and NSE $=89.35$; Leaf area: $\mathrm{RMSE}=$ $0.4583, \mathrm{MAE}=0.2647$ and NSE $=97.09$ ).

The approximate estimates of $E \circledast$ and $E(E)$ were obtained as 0.036 and 0.027 and the corresponding variances of mean growth rates were $1.5 \times 10-6$ and $2.12 \times 10-6$ ). These variances can be directly used as the bases of confidence intervals for the expected values, or for testing significance. Based on this, the growth analysis of plant was performed and the measures to increase the overall plant growth could be significantly estimated. This paper presents a crop growth estimation strategy without destructive sampling for tissue culture banana plantlet hardened in the greenhouse structure using BANN-based crop phenotypic model. These expected values of net assimilation rate and relative growth rate serves not only to predict the plant growth rate but also gives an avenue to provide a premise of crop growing in an ideal environment through comprehensive control of nutrient and energy consumption. Hence, the results can be used as an expert decision and multi-factors coordination control to improve the efficiency of banana tissue culture plantlet production in the greenhouse environment. 


\section{Acknowledgments}

The authors gratefully acknowledge the Director, ICARNational Research Centre for Banana, Tiruchirapalli and Indian Council of Agricultural Research (ICAR), New Delhi for the infra structural facility and financial for offered carrying out this study. Maintenance of plants and observations recorded by Senior TechnicalAssistant, Mr. M. Bathrinath is duly acknowledged.

\section{References}

Albright, L.D., R.S. Gates, K.G. Arvanitis and A.E. Drysdale: Environmental control for plants on earth and in space. IEEE Control Systems Magazine, pp. 28-47 (2001).

Carrol, M.S. and B.W. Dickinson: Construction of neural nets using the Radon transform. International 1989 Joint Conference on Neural Network, pp. 607-611 (DOI: 10.1109/IJCNN.1989.118639) (1989).

Chandra, S., R. Bandopadhyay, Vijay Kumar and R. Chandra: Acclimatization of cultured tissue plantlets: From laboratory to land. Biotechnology Letters, 32, 1199-1205 (2010).

Chernick, M.R. and R.A. Labudde: Revisiting qualms about bootstrap confidence intervals. American J. Math. Manag. Sci., 29, 437-456 (2009).

Cybenko, G.: Continuous valued neural networks with two hidden layers are sufficient. Technical Report. Department of Computer Sciences, Trfts. University, Medford, Mass (1988)

Cybenko, G.: Approximation by super positions of a sigmoidal function. Mathem. Cont. Signals Syst., 2, 303-314 (1989).

Efron, B. and R.J. Tibshirani: An introduction to the bootstrap. Chapman \& Hall/CRC, New York (1993).

Efron, B.: Bootstrap Methods: Another look at the Jackknife. The Annals of Statistics, 7, 1-26 (1979).

Fisher, N. I. and P. Hall: Bootstrap algorithms for small samples. J. Statistical Planning Infer., 27, 157-169 (1991).

Funahashi, K. I.: On the approximate realization of continuous mappings by neural networks. Neural Networks, 2, 183-192 (1989).

Goodwin, G. C. and K. S. Sin.: Adaptive Filtering Prediction and Control. Prentice-Hall Publishers, Englewood Cliffs, New Jersey (1984).

Hornik, K., M. Stinchcombe and H. White: Multilayer feedforward networks are universal approximators. Neural Networks, 2, 359366 (1989)

Kishor Kumar, M., R. Senthil Kumar, V. Sankar, T. Sakthivel, G. Karunakaran and P.C. Tripathi: Non-destructive estimation of leaf area of durian (Durio zibethinus) - An artificial neural network approach. Scientia Horticulturae, 219, 319-325 (2017).

Lin, F., X. Chen and H. Yao: Evaluating the use of Nash-Sutcliffe efficiency coefficient in Goodness-of-Fit measures for daily runoff simulation with SWAT. J. Hydro. Engin., 22, 5017023-5017029 (2017).

Max, J.F.J.: Greenhouse cover technology. Horticultural Reviews, 40, 259-396 (2012)

Mokhtarpour, H., B.S. Christopher, G. Saleh, A.B. Selamat, M.E. Asadi and B. Kamkar: Non-destructive estimation of maize leaf area, fresh weight, and dry weight using leaf length and leaf width. Communi. Biometry Crop Sci., 5, 19-26 (2010).

Nash, J.E. and J.V. Sutcliffe: River flow forecasting through conceptual models part I - A discussion of principles. J. Hydrology, 10, 282-290 (1970).

Perez, E. and C.R.R. Hooks: Preparing tissue-cultured banana plantlets for field planting. CTAHR Cooperative Extension Service Publication, BIO-8, p.3 (2008).

Pinter, J.P.J., J.C. Ritchie, J.L. Hatfield and G.F. Hart: The agricultural research service's remote sensing program: An example of interagency collaboration. Photogram. Engin. Remote Sen., 69, 615-618 (2003).

Pompelli, M.F., W.C. Antunes, D.T.R.G. Ferreira, P.G.S. Cavalcante, H. C. L. Wanderley-Filho and L. Endres: Allometric models for nondestructive leaf area estimation of Jatropha curcas. Biomass and Bioenergy, 36, 77-85 (2012).

Potdar, M.V. and K.R. Pawar: Non-destructive leaf area estimation in banana. Scientia Horticulturae, 45, 251-254 (1991).

Priddy, K.L. and P.E. Keller: Artificial Neural Networks: An Introduction. SPIE Press- The International Society for Optical Engineering, Bellingham, Washington, USA(2005).

Ronga, D., M. Zaccardelli, S. Lovelli, D, Perrone, E. Francia, J. Milc and N. Pecchioni: Biomass production and dry matter partitioning of processing tomato under organic vs conventional cropping systems in a Mediterranean environment. Scientia Horticulturae, 224, $163-170$ (2017).

Scholz, F. W.: The Bootstrap Small Sample Properties (edited version of a technical report of same title and issued as bestech-93-051). Boeing Computer Services, Research and Technology, University of Washington, Seattle, Washington (2007).

Serdar, U. and H. Demirsoy: Non-destructive leaf area estimation in chestnut. Scientia Horticulturae, 108, 227-230 (2006).

Sezer, I., F. Oner and Z. Mut: Non-destructive leaf area measurement in maize (Zea mays L.). J. Envir. Biol., 30, 785-790 (2009).

Simon H.: Neural networks: a comprehensive foundation, $2^{\text {nd }}$ Edn,. Pearson Prentice Hall (1999).

Slatyer, R.O. and S.A. Taylor: Terminology in plant- and soil-water relations. Nature, 187,922-924 (1960).

Sone, C., K. Saito and K. Futakuchi: Comparison of three methods for estimating leaf area index of upland rice cultivars. Crop Science, 49, 1438-1443 (2009).

Song, M.L., L.L. Zhang, W. Liu and R. Fisher: Bootstrap-DEA analysis of BRICS' energy efficiency based on small sample data. Applied Energy, 112, 1049-1055 (2013). 